\title{
Comparison between adherence assessments and blood glucose monitoring measures to predict A1c in type 1 diabetes
}

\author{
Gabriela Heiden Teló, Martina Schaan de Souza, Thaís Sturmer Andrade, Beatriz D’Agord Schaan \\ From 20th Brazilian Diabetes Society Congress \\ Porto Alegre, Brazil. 11-18 November 2015
}

\section{Background}

Treatment adherence is crucial in patients with diabetes; however, there is disagreement on how to measure adherence in adults with type 1 diabetes (T1D). Surveys have been validated to evaluate adherence, and several studies have demonstrated a strong correlation between frequency of blood glucose monitoring (BGM) and glycemic control.

\section{Objective}

We conducted multivariable regression analyses to compare adherence assessments and BGM measures with regard to their ability to predict A1c in adults with T1D.

\section{Materials and methods}

Four instruments evaluated adherence: Self-Care Inventory-Revised version (SCI-R), a self-administered survey; Diabetes Self Monitoring Profile (DSMP), a survey administered by trained researchers; a categorical (yes/no/ sometimes) self-report question ("In the past month, did you take care of your diabetes as your doctor recommended?"); and a continuous adherence self-evaluation, which ranged from 0-100. BGM frequency was evaluated by self-report, BGM diary, and meter download. Glycemic control was assessed by A1c (HPLC).

\section{Results}

Participants (N=82; 63\% males) were aged $39.0 \pm 13.1$ yrs. with a mean diabetes duration of $21.2 \pm 11.1$ yrs.; $27 \%$ had BGM frequency $>4$ times/day and 39\% were overweight/ obese. Mean A1c was $8.9 \pm 2.2 \%$ and only $11 \%$ met the target $\mathrm{HbA1c}$ of $<7 \%$. The adherence assessments appeared to be interrelated $(\mathrm{P}<0.01)$, as well as the BGM measures
$(\mathrm{P}<0.001)$. Among the adherence assessments, DSMP score was the strongest predictor of glycemic control $(\mathrm{r}=-0.32, \mathrm{P}=0.004)$, while BGM assessed by meter download was the strongest predictor of A1c among the BGM measures $(r=-40, P<0.001)$. Moreover, the correlation between DSMP score and BGM by meter download was the strongest identified correlation in the adherence and BGM measures $(r=0.52, P<0.001)$. All the self-report assessments had a significant but weak correlation with glycemic control $(\mathrm{r}=-0.27,-0.28 ; \mathrm{P} \leq 0.02)$. The final adjusted model identified the assessment of BGM frequency by meter download as the most robust predictor of A1c (estimate effect size $=-0,58, \mathrm{P}=0.003$ ). Demographics and clinical characteristics did not have an impact on the adherence-glycemic control relationship $(\mathrm{P}>0.05)$.

\section{Conclusions}

This study provided an opportunity to evaluate and compare adherence assessments to predict HbA1c. Although surveys like DSMP are an easy-to-use instrument to assess adherence, BGM assessment by meter download seems to have the strongest relationship with glycemic control in adults with T1D.

Published: 11 November 2015

doi:10.1186/1758-5996-7-S1-A170

Cite this article as: Teló et al:: Comparison between adherence

assessments and blood glucose monitoring measures to predict A1c in type 1 diabetes. Diabetology \& Metabolic Syndrome 2015 7(Suppl 1):A170.

* Correspondence: gabrielatelo@yahoo.com.br

UFRGS-Universidade Federal do Rio Grande do Sul, Porto Alegre, Brazil 